\title{
(3) Determination of minimum inhibitory concentration values (MICs) against Sporothrix brasiliensis and Sporothrix schenckii V.1
}

\author{
luanaborba ${ }^{1}$ \\ ${ }^{1}$ Universidade Federal do Rio de Janeiro \\ L. P. Borba-Santos
}

VERSION 1

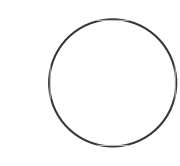

luanaborba

\section{OPEN ӘACCESS}

DOI:

dx.doi.org/10.17504/protocol s.io. bjfbkjin

Document Citation: luanabo rba 2020. Determination of minimum inhibitory concentration values (MICs) against Sporothrix brasiliensis and Sporothrix schenckii. protocols.io https://dx.doi.org/10.17504/p rotocols.i io. bjfbkjin

License: This is an open access document distributed under the terms of the Creative Commons Attribution License, which permits unrestricted use, distribution, and reproduction in any medium, provided the original author and source are credited

Created: Aug 06, 2020

Last Modified: Aug 06, 2020

\section{DOCUMENT integer ID:}

40131

Determination of minimum inhibitory concentration values (MICs) against Sporothrix brasiliensis and Sporothrix schenckii

The minimum inhibitory concentration (MIC) of compounds was determined using the broth microdilution technique described by the $\mathrm{CLSI}^{1}$ with modifications. 
1. Stock solutions of compounds in dimethyl sulfoxide (DMSO) at $1 \mathrm{mM}$ were diluted in RPMI 1640 medium $^{2}$ supplemented with $2 \%$ glucose and buffered to $\mathrm{pH} 7.2$, with $0.165 \mathrm{M}$ MOPS (from here on referred to as "supplemented RPMI") to obtain solutions 4-fold concentrated using sterile microtubes;

2. Itraconazole ${ }^{2}$ (reference antifungal) was also included in the experiment;

3. Aliquots of $100 \mu \mathrm{l}$ of compounds were added in two wells of the first column of a flat-bottom 96 -well microplate (KASVI, Brazil);

4. Aliquots of $100 \mu \mathrm{l}$ of supplemented RPMI were added in all microplate wells (including wells containing compounds) using a multichannel pipette and a serial 2-fold dilution was performed to the tenth column;

5. The eleventh column corresponded to the positive controls (containing $100 \mu$ l supplemented RPMI without compounds) and the twelfth to the negative controls (containing $200 \mu$ supplemented RPMI);

6. A standardized suspension of Sporothrix yeasts was adjusted using a Neubauer chamber, prepared in supplemented RPMI, and $100 \mu \mathrm{l}$ containing $2 \times 10^{5} \mathrm{CFU} / \mathrm{ml}$ was added in microplates containing compounds (except in the twelfth column);

7. The final concentration of compounds ranged from 0.002 to $1 \mu \mathrm{M}$, while yeasts were $1 \times 10^{5} \mathrm{CFU} / \mathrm{ml}$;

8. Samples were incubated at $35^{\circ} \mathrm{C}$ for $48 \mathrm{~h}$, in a $5 \% \mathrm{CO}_{2}$ atmosphere;

9. Fungal growth was analyzed by visual inspection in an inverted light microscope (Axiovert 100, ZEISS Company, Germany);

10. After visual inspection, samples were homogenized and the optical density was quantified by spectrophotometric readings at $492 \mathrm{~nm}$, in a microtiter plate reader (EMax Plus, Molecular Devices, USA);

11. The absorbance value for each well was subtracted from the mean value for the negative controls;

12. Inhibition of fungal growth (I) relative to positive controls was calculated according to the following equation: I = $100-(A \times 100 / C)$, where $A$ is the absorbance of treated wells, and $C$ is the absorbance of positive controls;

13. Concentrations that inhibit $50 \%$ and $80 \%$ of fungal growth $\left(\mathrm{IC}_{50}\right.$ and $\mathrm{IC}_{80}$, respectively) were estimated. 
${ }^{1}$ CLSI. Reference Method for Broth Dilution Antifungal Susceptibility Testing of Yeasts. 4th ed. CLSI standard M27.Wayne, PA: Clinical and Laboratory Standards Institute; 2017.

${ }^{2}$ Sigma Chemical Co., USA. 